\title{
DIAGNOSIS OF EDGE LOCALIZED MODE EVOLUTION IN DIII-D USING FAST-GATED CID AND INFRARED CAMERAS
}

\author{
by \\ M. GROTH, M.E. FENSTERMACHER, C.J. LASNIER, \\ R. HERNANDEZ, J.M. MOELLER, and R.A. STURZ
}




\section{DISCLAIMER}

This report was prepared as an account of work sponsored by an agency of the United States Government. Neither the United States Government nor any agency thereof, nor any of their employees, makes any warranty, express or implied, or assumes any legal liability or responsibility for the accuracy, completeness, or usefulness of any information, apparatus, product, or process disclosed, or represents that its use would not infringe privately owned rights. Reference herein to any specific commercial product, process, or service by trade name, trademark, manufacturer, or otherwise, does not necessarily constitute or imply its endorsement, recommendation, or favoring by the United States Government or any agency thereof. The views and opinions of authors expressed herein do not necessarily state or reflect those of the United States Government or any agency thereof. 


\title{
DIAGNOSIS OF EDGE LOCALIZED MODE EVOLUTION IN DIII-D USING FAST-GATED CID AND INFRARED CAMERAS
}

\author{
by \\ M. GROTH, ${ }^{\star}$ M.E. FENSTERMACHER, ${ }^{*}$ C.J. LASNIER, ${ }^{*}$ \\ R. HERNANDEZ, ${ }^{\dagger}$ J.M. MOELLER, ${ }^{*}$ and R.A. STURZ ${ }^{\ddagger}$
}

This is a preprint of a paper to be presented at the Fourteenth Topical Conference on High Temperature Plasma Diagnostics, July 8-11, 2002, Madison, Wisconsin, and to be published in the Proceedings.

*Lawrence Livermore National Laboratory, Livermore, California.

${ }^{\dagger}$ University of California, San Diego, La Jolla, California.

${ }^{\ddagger}$ Xybion Electronic Systems, San Diego, California.

\author{
Work supported by \\ the U.S. Department of Energy under \\ Contract Nos. DE-AC03-99ER54463, W-7405-ENG-48, \\ and Grant No. DE-FG03-95ER54294
}

GENERAL ATOMICS PROJECT 30033 AUGUST 2002 


\begin{abstract}
The tangentially viewing visible and vertically viewing infrared cameras systems on DIII-D were upgraded to permit emission measurements during edge localized modes (ELMs) with integration times as short as 1 and $100 \mu$ s respectively. The visible system was used to obtain 2-D poloidal profiles of CIII $(465 \mathrm{~nm})$ and $\mathrm{D}_{\alpha}(656.3 \mathrm{~nm})$ emission with $20 \mu$ s integration during various stages of ELM events in the lower DIII-D divertor. The infrared (IR) system was used to measure the heat flux to the divertor targets at $10 \mathrm{kHz}$ with $100 \mu \mathrm{s}$ exposure. Upgrades to the data processing and storage systems permitted efficient comparison of the temporal evolution of these measurements.
\end{abstract}




\section{INTRODUCTION}

Detailed physics measurements of the particle and energy pulses that propagate in the scrape-off-layer (SOL) and divertor of tokamaks, as a result of edge localized modes (ELMs), are needed for the development of techniques to mitigate the damage that these transients may cause to the target plates of future high power tokamaks. Depending on the operational regime, the energy released during an ELM event can be as high as 50\%

of the electron energy in the H-mode pedestal region. ${ }^{1}$ The deposition of this energy onto the target plate during a time less than a millisecond can lead to divertor surface ablation, unacceptable target plate erosion, and significantly reduced target life time. ${ }^{2}$

To characterize the effect of ELMs in the SOL and divertor of DIII-D, recent hardware upgrades were done to obtain sub-millisecond integration time from two existing TV camera systems that measure the line emission in the visible wavelength range, ${ }^{3}$ and the target surface temperature in the infrared (IR) range. ${ }^{4}$ The former measurement surveyed the spatial distribution of the deuterium neutrals and carbon ions, thus describing the effects of ELMs on the divertor plasma particles; the latter measurement enables us to calculate the heat flux to the target plates due to ELMs. These upgrades and first results obtained with the new hardware are described in this paper. 


\section{HARDWARE AND SOFTWARE UPGRADES}

\section{A. Visible Camera Upgrades}

Substantial hardware upgrades were made, since the most recent report, ${ }^{5}$ to the visible camera system used for measurements of deuterium and impurity line emission during ELMs. As previously, the lower divertor in DIII-D is viewed tangentially using a mirror mounted inside the tokamak vacuum vessel. However, in the new system the image is relayed out of the tokamak by a set of optics (Fig. 1), that replaced the fiber image guide of the previous arrangement, thus permitting an absolute calibration of the system. The optical relay design is similar to that used for VUV imaging on DIII-D. ${ }^{6}$ Remotely selectable filters were inserted into the optical path to isolate emission from a single spectral line; the CIII (465 nm) line from beryllium-like carbon and the $\mathrm{D}_{\alpha}$ emission $(653.6 \mathrm{~nm})$ were used in the ELM measurements. Two-dimensional profiles of the line emission during ELMs were inferred from the 3-D tangential raw data using tomographic reconstruction techniques. ${ }^{5}$ The spatial resolution of the raw datais approximately $0.5 \mathrm{~cm}$. Resizing the raw data to a manageable array of (640x240) elements resulted in an overall spatial resolution of $2 \mathrm{~cm} /$ pixel in the 2-D solution. The routine SBOLS of the SLATEC Common Mathematical Library ${ }^{7}$ was adapted to perform the least-square regression, constraining the solution to non-negative values. An alignment of each image on a background fiducial was still necessary due to slight translational and rotational displacement of the camera relative to the most recent spatial calibration.

The emission during ELM events was obtained with a regular CID camera (CIDTEK $3710 \mathrm{D})^{8}$ equipped with an intensifier (DELFT ELECTRONIC PRODUCTS $)^{9}$ to achieve the desired time resolution of the order $1-100 \mu \mathrm{s}$ at sufficient light intensity. The intensifier acts as a fast shutter, photon current converter and amplifier. The intensifier system (GEN II) can be divided in the

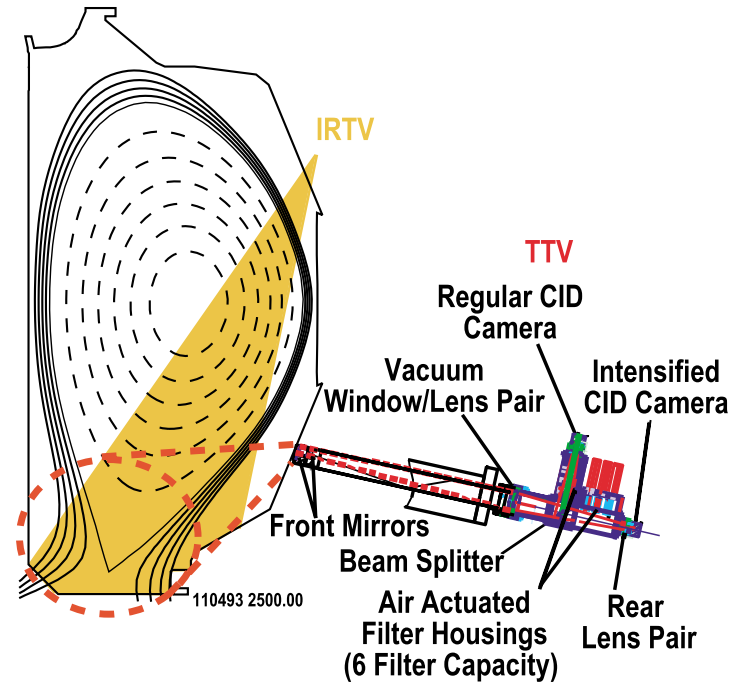

Fig. 1. Field-of-view and hardware of the tangential TV system (TTV) and field-of-view of the infrared PHOTON system (IRTV). 
three principal parts photocathode/MCP, phosphor screen, CID imager (Fig. 2). The phosphor screen, which replaces the anode in intensifiers, converts the electron current back into a photon current, which is then focused onto the CID imager using a glass taper. The photocathode material is a multi-alkali substance with a broad spectral response between 400 and $800 \mathrm{~nm}$. The MCP used allows integration times between $300 \mathrm{~ns}$ and $33 \mathrm{~ms}$, with gate rise times as low as $1 \mathrm{~ns}$. The signal amplification of the intensifier used was of up to 4 orders of magnitude, which was established independently in side lab tests. the gain settings on the camera control unit. The phosphor screen (P20) decays at a the gain settings on the camera control unit. The phosphor screen (P20) decays at a characteristic time scale of $10-100 \mu \mathrm{s}$, depending on the intensity of the image and the exposure time. This time is well below the intra-gate dwell time of $16.6 \mathrm{~ms}$, ensuring that there is no aliasing from one image to the next. The time response of the photocathode and the MCP, as well as the delay time of the gate signal due to $50 \mathrm{~m}$ of cables between the camera head and control units was well below $1 \mu$ s. The image was read out from the CID imager as a 2:1 interlaced RS-170 video signal that was both recorded to video tape and digitized for analysis.

During the experimental campaign reported here, the intensified camera was triggered internally by its control unit. The present images are therefore random snapshots of the emission, which needed to be sorted with respect to the ELM onset time as observed by other diagnostics. In the future campaigns, we plan to trigger the camera on other, submillisecond fast diagnostics, e.g. on midplane photodiodes. A triggering control unit has been developed that will be armed by an input signal from such an external diagnostic. It then takes full control of the intensified camera triggering, at either constant or cumulative time steps. If ELMs, that are spaced in time with an accuracy of milliseconds, can be produced, then this experimental method will increase the number of images that can capture part of the ELM cycle compared to internal triggering.

The recording, processing and storage of the video images were handled by National Instruments (NI) ${ }^{10}$ hardware and software (Fig. 3). The image was put through a time/date annotator and captured in real time at 60 fields/second by a frame grabber (NI PCI-1407). During the plasma discharge the video data was stored in RAM of a PC, a $5 \mathrm{~s}$ 
TTV ICID System

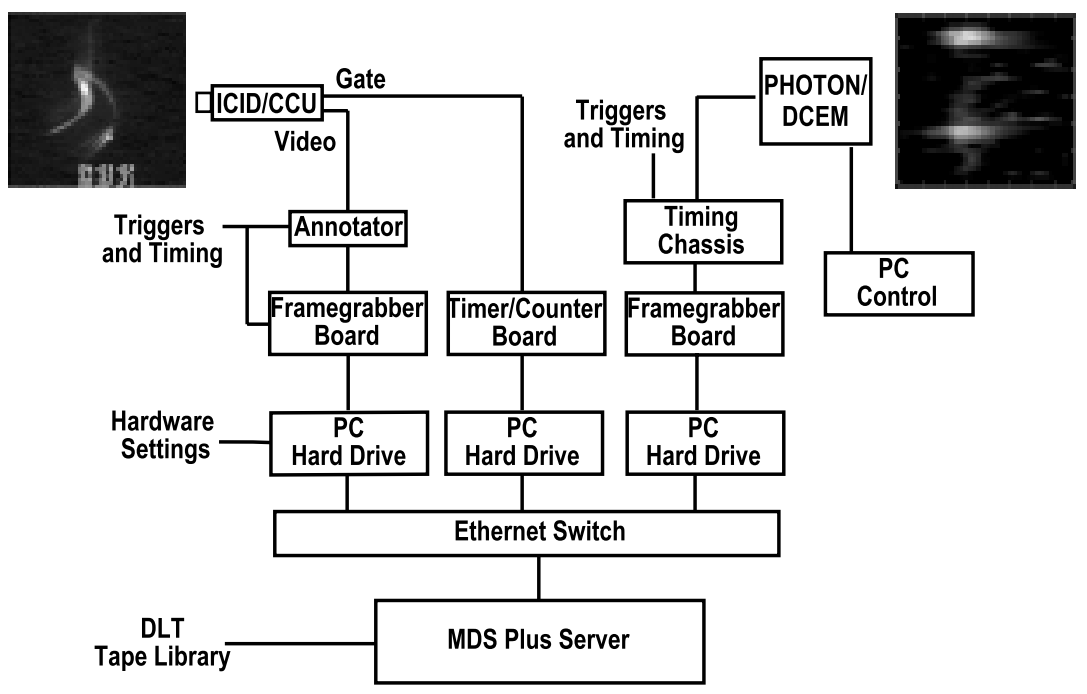

Fig. 3. Overview of the new data acquisition system of the ICID TTV and IRTV PHOTON cameras.

long pulse requiring about $46 \mathrm{MB}$ of memory. LabView based software was used to manage the subsequent image processing, visualisation, intermediate storage on the PC hard disk, and the transfer to a MDSPlus data storage server between plasma discharges. This has the great advantage over the storage on high-quality VHS tape used previously, since the image data are readily retrievable in digital format. The digitized video data can be viewed immediately after the plasma discharge using the same software. For backup reasons we also maintained the established data storage on VHS tape. The gate timing, accurate recording of which is crucial to this study, was acquired by a NI PCI-6602 counter/timer operated in its "buffered semiperiod" mode. Rising and falling gate edges were timestamped by an $80 \mathrm{MHz}$ clock, relative to an arbitrary start time. These values were tied to the DIII-D diagnostics trigger by another counter that measured the time interval to the first gate after the $\mathrm{t}=0$ trigger. From these measurements gate times and gate widths relative to the shot cycle were derived to a precision of $12.5 \mathrm{~ns}$. The collection of gate and dwell times was handled by LabView software and transferred to a MDSPlus server after each plasma discharge.

\section{B. Upgrades to the Infrared PHOTON Camera System}

Sub-millisecond fast infrared thermography was used at DIII-D to measure the transient ELM heat pulse loads onto the target. The infrared detector used was a $128 \times 128$ pixel element, InSb focal plane array provided by AMBER ENGINEERING, ${ }^{11}$ and assembled with the necessary digital control electronics by PACIFIC ADVANCED 
TECHNOLOGY. ${ }^{12}$ The system will hereafter be referred to as the PHOTON camera. The sensor head module was mounted on a "cold plate" which was cooled by liquid nitrogen, and shielded against neutrons by a $10 \mathrm{~cm}$ thick layer of borated polyethylene. The sensor head supplies the focal plane with necessary clocks as well as performing a 12-bit analogto-digital conversion of the output video. There are four output channels, each operated at a conversion rate of 5 million pixels per second. A PC provided the control of the camera electronics (Fig. 3).

The PHOTON camera was run in "line pair mode", permitting 9900 frames/s to be read out. The exposure time of the camera was software-controlled and typically set to 1-20 $\mu$ s. The time was recorded by replacing part of the pixel top row with an encoded time counter, that was generated using a DIII-D $1 \mathrm{MHz}$ clock and the $\mathrm{t}=0$ trigger. A frame grabber (NI PCI-1424) captures the digital signal. Spatially, line pair mode suppressed all other pixels of the array, except two, user-selected contiguous horizontal lines. Alternatively, the full 2-D image can be obtained with the camera set to "image mode", at 267 frames/second. The data was initially stored locally on a PC hard disk, and transferred after the plasma discharge to the MDSPlus server.

The field-of-view of the PHOTON camera is shown in Fig. 1. The infrared photons $\left(\lambda_{I R} \sim 3-5 \mu \mathrm{m}\right)$ were relayed through a vessel viewport $(\mathrm{ZnSe})$, an objective lens, a field lens, a close-up lens, and finally focused onto the focal plane by a telescope. The field-ofview allowed a full image of $80 \times 100 \mathrm{~cm}$ divertor target with a spatial resolution of $1.11 \mathrm{~cm} /$ camera pixel.

Absolute calibration of the PHOTON camera was achieved by comparing the signal level with the target temperature as measured by thermocouples during a tokamak bake. Since the maximum temperature achieved during the bake is $650 \mathrm{~K}$, it was necessary to extrapolate (square-root fit) to higher temperature seen during plasma discharges and certainly during ELMs. From the thermal properties of the ATJ graphite tiles, the heat flux was calculated from temporal changes in surface temperature. 


\section{IMAGE DATA INTERPRETATION AND RESULTS}

The RS-170 video output format of the CIDTEC 3710D imager operated in an interline frame transfer mode, and when operated with the fast gated intensifier in these experiments produced images of the same ELM event on two successive fields of digitized data. Video frames played back on RS-170 TV monitors have even and odd fields 2:1 interlaced to make a frame every $33 \mathrm{~ms}$. The individual fields represent charge accumulated on the imager for $33 \mathrm{~ms}$ and then readout in $16.7 \mathrm{~ms}$ to make up the video signal (Fig. 4). In the ELM experiment the overlap of a bright ELM event with the short $(20 \mu \mathrm{s})$ intensifier gate-on interval occurred with low frequency $(50-100 \mathrm{~Hz})$. One of these isolated events occurring in the even field of frame 1 also was recorded in the odd field of the following frame. Thus, each ELM that was imaged by the camera appeared in at least two digitized fields. If two ELM events were imaged during two successive intensifier gate-on intervals then three successive fields showed increased brightness. The first of these is the image of the first ELM, the last is the image of the second ELM and the middle field is the superposition of the two ELMs. No other aliasing of the images occurred as long as the gate-on interval was short compared with the $16.7 \mathrm{~ms}$ field readout time.

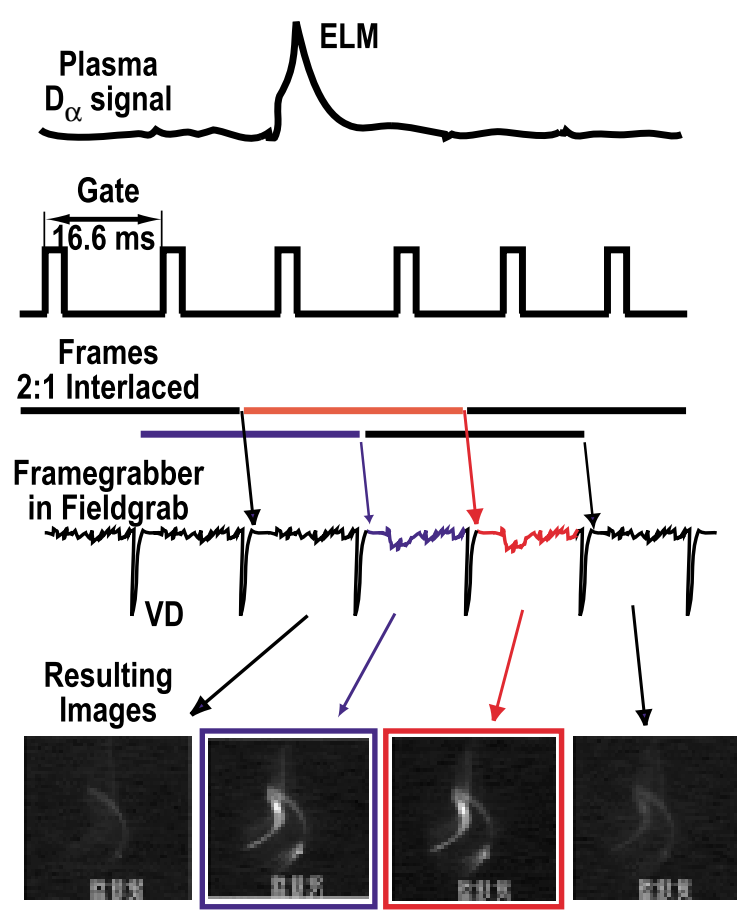

Fig. 4. Gated, image-intensified CID camera in interline frame transfer mode: Event, gate, frame architecture, readout, field grab, and resulting image. Due to interline frame transfer one event appears on two successive fields. 
Figure 5 shows two examples of the raw 3-D image data of CIII emission (a, c) in ELMy H-mode discharges, and their 2-D reconstruction on a poloidal plane assuming toroidal symmetry $(b, d)$. These particular data shown were obtained without the precise knowledge of the gate time relative to the ELM onset as observed by other diagnostics. The CIII emission profiles are significantly different and can be interpreted as an attached [Fig. 5(b)] and detached divertor [Fig. 5(d)]. Modeling of ELMs using B2/EIRENE indicated that during the deposition of the ELM heat pulse the divertor electron temperature rises, which moves the CIII radiation closer to the target [Fig. 5(b)]. ${ }^{13}$ With the arrival of the ELM particle pulse, the divertor plasma in the model transiently detaches, so that the CIII emission is expected to be around the X-point [Fig. 5(d)].

\section{DIII-D Shot 107444, CIII}

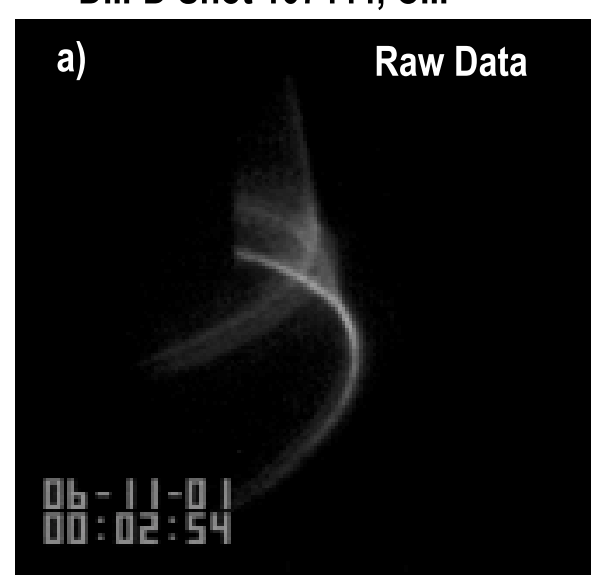

\section{DIII-D Shot 107444, CIII}

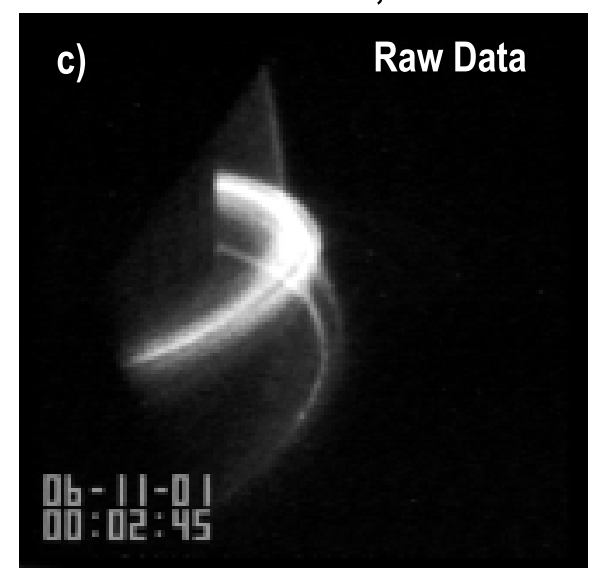

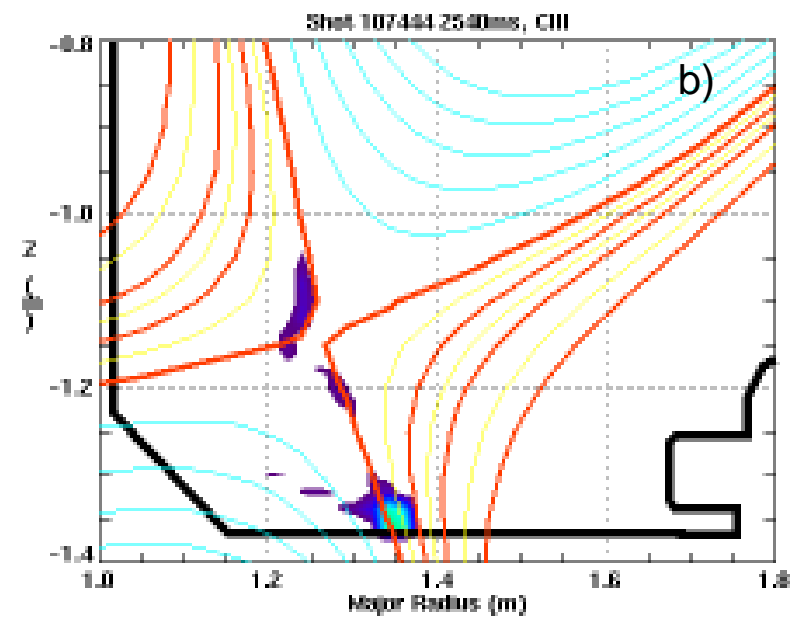

0

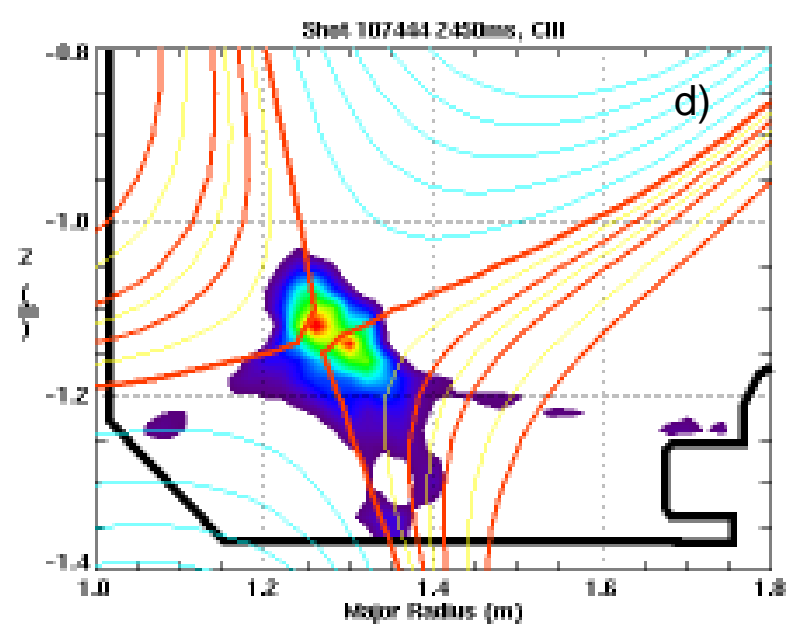

Fig. 5. Variation of the CIII emission during the ELM evolution. The 3-D image data (a,c) were taken at times during the ELM cycle. The reconstructed 2-D poloidal emission profiles (b,d) imply an attached divertor plasma (b) and a detached divertor plasma (d). The blue, yellow, and orange lines represent the magnetic flux surfaces; the orange field lines are spaced $1 \mathrm{~cm}$ at the outer midplane. 
Figure 6 gives an example of the target heat profiles obtained from the PHOTON camera during a type-I ELMy H-mode discharge. The heat due to an ELM is deposited onto the target plate within $400 \mu \mathrm{s}$, with a high degree of in/out symmetry.

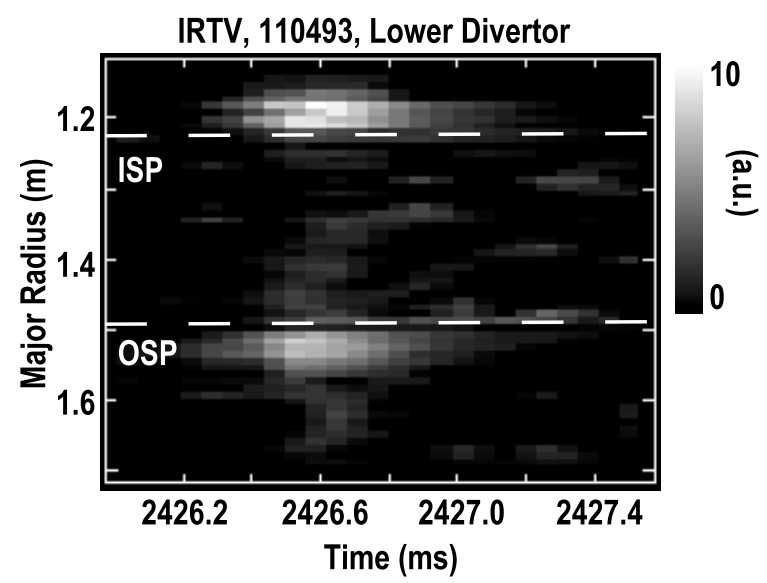

Fig. 6. Example of 2-D target heat flux profiles obtained during a type-I ELM measured by the PHOTON camera. The acronyms ISP and OSP denote the inner and outer strike points, respectively. 


\section{REFERENCES}

${ }^{1}$ A.W. Leonard et al., J. Nucl. Mater. 290-293, 1097 (2001).

${ }^{2}$ H.D. Pacher, "Disruption and ELM Erosion," App. E9, Sec. 1.7 (Divertor), ITER Design Description Document ITER No. G17 DDD 1 96-08-21 W2.1 August 1996.

${ }^{3}$ M.E. Fenstermacher et al., Phys. Plasmas 4, 1761 (1997).

${ }^{4}$ D.N. Hill et al.; Rev. Sci. Instrum. 59, 1225 (1988).

${ }^{5}$ M.E. Fenstermacher et al., Rev. Sci. Instrum. 68, 974 (1997).

${ }^{6}$ D.G. Nilson et al., Rev. Sci. Instrum. 70, 738 (1999).

${ }^{7}$ http://www.kachinatech.com/ hjjou/slatec-doc/.

${ }^{8}$ CID Technology Inc. (CIDTEC), 101 Commerce Blvd, Liverpool, NY 13088, USA, http://www.cidetec.com/.

${ }^{9}$ Delft Instruments NV, Röntgenweg 1, 2624 BD, P.O. Box 103, 2600 AC Delft, The Netherlands, http://www.delftinstruments.com/dep/dep.htm.

${ }^{10}$ National Instruments Corporation, 11500 N Mopac Expwy, Austin, TX 78759-3504, USA, http://www.ni.com/.

${ }^{11}$ Raytheon - Infrared, 4105 Tami Lane, Central Point, OR 97502, USA, http://www.raytheon.com/.

${ }^{12}$ Pacific Advanced Technology, 1000 Edison St., P.O. Box 359, Santa Ynez, CA 93460-0359, USA, http://patinc.com/.

${ }^{13}$ J.T. Hogan et al., "Modeling of ELM Events and Their Effect on Impurity Enrichment," Proc. 15 ${ }^{\text {th }}$ Int. Conf. on Plasma Surface Interaction, Gifu, Japan (2002). 
DIAGNOSIS OF EDGE LOCALIZED MODE EVOLUTION IN DIII-D

\section{ACKNOWLEDGMENTS}

This work was performed under the auspices of the U.S. Department of Energy under Contract Nos. DE-AC03-99ER54463, W-7405-ENG-48, and Grant No. DE-FG0395ER54294. The authors would like to thank R. Ellis for his invaluable effort in supporting diagnostic work. 